\title{
Exkurs zur Waffe
}

Eine genaue Lektüre legt also mitunter offen, wie einer Waffe - mit Latour - Handlungsfähigkeit eignet; menschliche Intentionalität wird durch dingliche Vermittlungsleistung kontaminiert und der hybride, der Fetischcharakter der Versammlung von Subjekt und Artefakt ersichtlich. So wird die sakrosankte Grenze zwischen Mensch und Ding überschritten, was in Abschnitt 4 der Einleitung als abstrahiertes Merkmal zahlreicher Fetischbegriffe eingeführt worden war.

Aus Perspektive dieser Diskurse, das heißt: mit dem Blick des Fetischjägers und Idolenzertrümmerers, gäbe es gerade über Waffen viel zu sagen. In keinem anderen Bereich ist Fetischisierung so naheliegend wie hier; nirgendwo scheint die Verkörperung von Handlungsmacht so greifbar, bei gleichzeitig nicht immer und nicht jedem transparenter Funktionsweise. Kaum ein Gegenstand ist so offensichtlich zur Ermächtigung seines Besitzers geeignet. Historisch überlagern sich in den Projektionen von Fetischisten und Antifetischisten nicht selten Fetischund Waffencharakter des materiellen Objekts. In der Wahrnehmung der europäischen Kolonisatoren dienten zahlreiche afrikanische Fetische einem waffenähnlichen Zweck, darüber hinaus wurde auch von den >Wilden keine Unterscheidung zwischen den Waffen und den religiösen Artefakten der Europäer kennen. Der Fetischismus in der Sexualpathologie spricht metaphorisch, aber eben nicht nur metaphorisch, von >Waffensammlungen zum Schutz vor der Anerkennung der weiblichen Kastration. ${ }^{1}$ Womöglich überrascht also der Befund, dass Hochgatterers Texte dem so naheliegenden Waffenfetisch nur wenig Aufmerksamkeit widmen.

Dieses Kapitel geht, ebenso wie das zum Kunst-Fetisch, einer im Werk des Autors ausbleibenden Fetischisierung nach. Es wirft dabei weniger die Frage nach dem ,Warum auf, sondern zeichnet vielmehr in aller Kürze nach, wie weit die betreffenden Dinge verschiedenen Fetischkonzepten entsprechen, wo sie von diesen abzweigen - und wohin.

1 Vgl. Kohl, Die Macht der Dinge (2003), S. 24; Pietz, The Problem of the Fetish, II (1987), S. 41; Smirnoff, Die fetischistische Transaktion (1972 [1970]), S. 103. 
So erfährt die Ding-Gattung >Waffe hier anderweitig eine durchaus bemerkenswerte Behandlung. Sie zerfällt in Hochgatterers Texten vorderhand in zwei Kategorien: Es gibt Waffen, die ganz ihrem Skript gemäß verwendet und so zum Glied in einer fatalen Ereigniskette werden, die also - mit Latour - »attachments [...] that kill ${ }^{2}$ darstellen (das Gewehr in Über Raben, der Totschläger in der Kurzen Geschichte, eine Steinschleuder in Fliege fort, fliege fort). Dagegen gibt es auch Waffen, die nicht ihrem Zweck gemäß eingesetzt werden - und dennoch eine Art von Wirkung zeigen. Die Wirksamkeit dieser zweiten Kategorie wird aus fiktiven oder imaginären Kontexten gespeist. Die genauere Betrachtung zweier Vertreter dieser Kategorie legt letztlich - am unerwarteten Ort - ein Plädoyer für die Fiktion, die emanzipative Kraft der erzählenden Künste frei.

Setzen wir aber zuerst beim bis dato jüngsten Roman des Autors an, bei Fliege fort, fliege fort (2019). Der voraussichtliche Abschluss der Furth-Trilogie hält gleich eine ganze Reihe von Waffen für die gegenwärtige Fragestellung parat, die die Differenz zwischen real verwendeter und imaginär aufgeladener Waffe zeigen.

Auf zwei Handlungsebenen erzählt der Roman von einer Reihe von Verbrechen in der Handlungsgegenwart in der fiktiven Kleinstadt Furth - Vandalismus, Gewalt gegen Seniorinnen und Senioren, nicht zuletzt eine Kindesentführung -, zum anderen von Geschehnissen, die sich in der nicht allzu fernen Vergangenheit in einem Kinderheim abgespielt haben: Das Regime der Heimleitung beruhte dort auf brutalen Disziplinarmaßnahmen. Am obersten Ende einer Stufenleiter ritualisierter Gewaltakte stand dabei der sogenannte ১Siegelring`: ein Faustschlag, verübt vom Direktor des Heims, Richard Schnabel, dessen Siegelring dabei die Kopfhaut des Kindes aufriss. "Jetzt kriegst du den Siegelring, du wirst ihn überleben« (FF 245), so lautet dazu der Stehsatz des Handlangers. >Den Siegelring kriegen $<$ Diese Formulierung erhält sich über die Jahrzehnte auch in der Familie des Direktors. Er gibt die Formulierung - ihres realen Zusammenhangs entkleidet - an seine Enkelkinder weiter, und wir hören den Satz aus dem Mund seines Enkelsohnes, während einer Befragung durch die Polizei. Seine Schwester ist in der Gegenwart der Romanhandlung Opfer einer Entführung geworden, »und wenn sie wieder da ist «, so die Rachephantasie ihres siebenjährigen Bruders, »kriegt der Entführer den Siegelring.« (FF 122)

Darüber hinaus wird der Ring noch eingehend beschrieben, seine Herkunft wird kurz referiert (FF 123), jedoch bleibt der Ring auf Handlungsebene frei von weiteren Zuschreibungen (von Wert, Handlungsmacht etc.). Er trage ihn nur noch aus Gewohnheit, so Schnabel (ebd.). Stattdessen wird der Ring ${ }^{3}$ Scharnier des kri-

2 Latour, Factures/fractures (1999), S. 30.

3 Dessen Ikonographie sich im Text übrigens auch - diesmal durchaus identitätsstiftend - auf dem Emblem eines rechtsradikalen Sicherheitsdienstes wiederfindet. (FF 161) 
minalistischen Plots. An der Formulierung >den Siegelring bekommen ne sprachliche Mehrdeutigkeit ihren Ausgangspunkt, die - wie auch andernorts im Werk Hochgatterers - die Unterschiedenheit der Codes aufzeigt, die in den Romanen des Autors parallel geführt werden: Die Polizisten missdeuten nämlich diese Formulierung ganz grundlegend, wenn sie zu verstehen meinen, dass der Bub dem Entführer den Ring als Lösegeld anbieten will. ${ }^{4}$

Aus Perspektive der Leserinnen und Leser gibt es zudem einen intertextuellen Verweis, der vom Ring ausgeht und einen Hinweis auf den Täter und die Umstände seiner Täterschaft liefert: Früh im Text bringt der Benediktinerpater Bauer einen Stapel DVDs in das städtische Jugendzentrum:»[E]igentlich habe er einen gemeinsamen Filmabend vorschlagen wollen - Der Herr der Ringe, er habe alle drei Teile mitgebracht.« (FF 21) Der Titel von J. R. R. Tolkiens Trilogie (bzw. hier Peter Jacksons Verfilmung) ist ein Hinweis auf den Heimleiter (als $>$ Herrn des Rings $\triangleleft$ ), dessen frühere Untaten den Hintergrund der Entführung seiner Enkeltochter bilden: Das Motiv dieser Entführung nämlich ist Rache im Namen der damaligen Opfer. Zudem ist der titelgebende Ring bei Tolkien mit der Kraft ausgestattet, seinen Träger unsichtbar zu machen. Wenn nun der ehemalige Heimleiter gegenüber der Polizei über seinen Ring sagt, »[e]r habe ihn vor Jahrzehnten verliehen bekommen « und "[e]r sei damals Vorsitzender eines Vereins gewesen, der sich in der Region sozial engagiert habe« (FF 123), dann ist damit auf das Sozialprestige, auf eine hierarchische und gesellschaftliche Position hingewiesen, hinter der Täter und Taten unsichtbar werden.

Die Misshandlung durch den Siegelring und das um diesen gesponnene Ritual werden im Verlauf der Handlung nachgeahmt: Eine namenlos bleibende ehemalige Erzieherin ebendieses Heims entführt nicht nur die Enkelin des ehemaligen Direktors, sondern wiederholt die verschiedenen Bestrafungsrituale, die den Heimalltag ausgemacht haben, an den damaligen Mittätern, an Erziehern und einer geistlichen Schwester. Einer der Handlanger des Direktors wird in der Handlungsgegenwart des Romans im Auftrag der ehemaligen Erzieherin überfallen, auf die Knie gezwungen, dann wird seine Kopfhaut gespalten (FF 257) - mithilfe nicht eines Rings, sondern eines Dolches. Auf diesem Dolch treffen sich zwei Perspektiven, die den erwähnten Gegensatz, die zwei Kategorien illustrieren, in die die 
Tatwaffen dieser Texte fallen: Sie werden entweder fetischisiert oder als Waffen gebraucht, interessanterweise nicht beides zugleich. Der Dolch geht durch mehrere Hände, und ursprünglich trägt er die Merkmale des Fetischs fast offensichtlich: Hüssein, ein junger Flüchtling, stiehlt ihn bei einem Waffenhändler. Der Dolch sehe aus, als stamme er aus dem Irak, der ehemaligen Heimat des jungen Mannes; ihm dient er als materieller Bürge der eigenen Identität. Aus der Perspektive der anderen Protagonisten natürlich ist das ein recht untauglicher Bürge, immerhin handelt es sich um Massenware "made in Pakistan« (FF 81), um Folklore-Kitsch, der somit für Herkunft und Identität gerade nicht einstehen kann. In dieser hybriden Herkunft - ein pakistanischer Dolch nach irakischem Muster, geklaut im österreichischen Waffenhandel - und in dem von seinem Besitzer paraphrasierten »Ich weiß zwar, dennoch aber ...« spiegeln sich Muster des Fetischs wie des Fetischismus, wie sie im antifetischistischen Diskurs geprägt wurden. Der Dolch jedoch wechselt den Besitzer, und damit die Funktion; die fetischistischen Anklänge verschwinden, im neuen Zusammenhang wird aus dem Dolch ein Werkzeug, nämlich eine Tatwaffe.

Hüssein ist Bewohner eines Heims für unbegleitete minderjährige Flüchtlinge, das in den Mauern des ehemaligen Kinderheims untergebracht ist, was im Roman eine historische Kontinuität der Kasernierung und Misshandlung Schutzbefohlener sichtbar macht. Während einer Razzia durch einen rechtsradikalen Sicherheitsdienst und die (fiktive, aber nicht allzu fiktive) »Sondereinheit Ausreise« der Polizei wird der Dolch zuerst versteckt und dann, nachdem sein Besitzer misshandelt und abtransportiert wird, von dessen Zimmerkollegen Malik außer Haus gebracht. Malik ist nun einerseits mit dem Sohn einer der Hauptfiguren des Romans befreundet, mit Tobias, dem Sohn des Psychiaters Horn. Malik ist aber auch Gast im Jugendzentrum und möglicherweise Mitglied der Gruppe Jugendlicher, die im Auftrag der namenlosen ehemaligen Erzieherin die Racheakte an der früheren Belegschaft des Kinderheims verüben. Dass Malik an diesen Taten wirklich Anteil hat, ist unwahrscheinlich; wahrscheinlicher, dass er den Dolch dazu weitergegeben hat. Davor taucht dieser noch einmal im Haus des Psychiaters auf, worauf ein Gespräch zwischen Vater und Sohn schließen lässt (FF 162).

Der Dolch dient hier nicht mehr zur Identitätsstiftung. Stattdessen zeichnet er das weitreichende und teils bis zum Schluss im Dunkeln bleibende Netzwerk nach, das aus Jugendlichen sowie Menschen am Rand der gesellschaftlichen Wahrnehmung besteht und das der Aufmerksamkeit sämtlicher Ermittler entgeht. Dieses Netzwerk macht, so problematisch seine Taten im Einzelnen sein mögen, die Utopie im Kern dieses ansonsten so düsteren Romans aus. Gegen eine Welt der resignierten, apathischen oder verantwortungslosen Amts- und Meinungsträger steht, in Form dieses den Roman infiltrierenden, aber unbemerkt bleibenden Bündnisses, eine Utopie von Solidarität. 
Mit dem Revolver in Caretta Caretta (1999) wird ein wenig gedroht, aber niemand erschossen. Darüber hinaus und hauptsächlich hat er seinen Platz in dem das Buch abschließenden improvisierten Begräbnisritual. Der dabei abgegebene Schuss hat ausschließlich symbolische Bedeutung. Davor hingegen wurde der Revolver schon explizit und reichhaltig >besprochen`, mit Bildern und Tagträumen assoziiert.

Er taucht auf, als der jugendliche Protagonist Dominik sich auf einer Schwarzfahrt im Gepäckfach eines Zugabteils versteckt und das Gespräch zweier Fahrgäste belauscht. Von Anfang an tritt an der Waffe nicht der technische Gegenstand, sondern der mit der Waffe am engsten assoziierte Text - ihr Name - und ein damit verbundener Komplex an Motiven und Handlungsmomenten in den Vordergrund: Es handelt sich um einen Colt Anaconda (CC 21); dass die Herstellerbezeichnung zu Beginn unterschlagen wird, lenkt die Assoziation Dominiks ins Tierreich: »Die Anaconda, fiel mir ein, tötet ihre Opfer, indem sie sie erdrosselt. Sie frißt kleine Kinder und Hängebauchschweine, jeweils am Stück. Sie kann dazu ihre Kiefer ausrenken.«(CC 18f.) Dominik wird entdeckt; die beiden amüsierten Fahrgäste führen seine Assoziation zu zweit weiter:

»Die Anaconda«, schnarrt schließlich der Kleinere, »dieses Ding, das den Nilpferdmüttern ihre Jungen wegfrißt?«

$[\ldots]$

»Nilpferdmütter«, brüllt der Große, »die armen Nilpferdmütter!«

»Happ macht die Anaconda und weg ist das Nilpferdkind!«

»Wie sie dann weint, die Nilpferdmutter!«

»Die Anaconda schwimmt davon und sieht aus, als habe sie ein Faß verschluckt.« (CC 20)

Über den Umweg des Tiernamens bekommt die Waffe in diesem >gemeinsamen Tagtraum ^ die Fähigkeit zugesprochen, Müttern ihre Kinder zu nehmen; bald darauf übernimmt Dominik das Motiv und imaginiert eine entsprechende Szene: »Ich denke daran, wie so ein nettes Tier den Unterkiefer lockert und sich selbst den Happen vors Maul plaziert, kopfvoran und ohne dem armen Kind vorher das T-Shirt auszuziehen oder das kleingeblümte Kleid mit Kragen." (CC 30)

In der kollektiven Imagination Dominiks und der beiden Passagiere vollführt der Revolver/die Anaconda den gewaltsamen Einbruch in die Einheit aus Mutter und Kind, wie ihn Dominik aus eigener Erfahrung kennt. Das Prinzip des Vaters, wie es uns aus der Psychoanalyse und Dominik handgreiflich durch seinen Stiefvater bekannt ist, erscheint in Gestalt einer Faustfeuerwaffe. Dominik stiehlt die Waffe, und in der Assoziationslogik des Textes bedeutet das: Dominik eignet sich diese quasi-väterliche Macht des Kinderfressens und Mütterberaubens an. Immer wieder schwelgt er von nun an in der Vorstellung, unliebsame Zeitgenossen zu bedrohen oder zu erschießen (CC 25, 40). 
Zum Einsatz kommt die Waffe aber erst am Schluss, als Teil eines Rituals, in dem eine Vaterfigur beigesetzt wird: Kossitzky, dem im Verlauf des Romangeschehens unerwartet positiv besetzten Freier Dominiks, wird nach seinem Tod eine improvisierte Beerdigung zuteil. In deren Verlauf wird der Revolver abgefeuert, dabei aber zeigt sich die väterlich konnotierte Macht des Revolvers als überraschend gering: Der Rückstoß, vor dem die beiden Gestalten im Zugabteil noch gewarnt hatten, fällt schwächer aus als gedacht (CC 219). Zudem wird die Entwicklung, die Dominik im Verlauf des Romans genommen hat (vgl. dazu eingehender Kap. III.8), auch am Einsatz der Schusswaffe ersichtlich: Dominik hat sich die Waffe und die mit ihr motivisch verknüpfte Macht nicht angeeignet (um sie wiederum selbst anzuwenden), er hat sie sich anverwandelt. Den zahlreichen realen Gewaltakten tritt kein weiterer entgegen. An Stelle der Identifikation mit dem Vater steht die Verabschiedung seines zweifelhaften Erbes.

Vergleichbares gilt für ein Schwert im Roman Das Matratzenhaus (2010): Es wird durch einen Intertext in den Roman getragen, dort langwierig hergestellt, um dann jedoch nur in einer auch im Romangeschehen fiktiven Binnenhandlung zur Anwendung zu kommen.

Seine Entstehung verdankt es der Bekanntschaft der Protagonistin Fanni mit Quentin Tarantinos zweiteiligem Filmwerk Kill Bill (der »Film in der gelben und roten Hülle« - MH 119). ${ }^{5}$ Fanni will ihren Adoptivvater ermorden - als Rache nicht für den selbst erlebten Missbrauch, sondern für die Ermordung ihrer Adoptivschwester Switi (im Zuge der Herstellung eines Snuff-Films). Die von Fanni geplante Tat und ihre rituellen Aspekte sind dabei auf mehrere Motive des Tarantino-Films zurückzuführen. Dieser, eine Collage aus B-Movie-Anleihen, behandelt den Rachefeldzug einer Auftragsmörderin gegen ihren ehemaligen Vorgesetzten und Geliebten Bill. Die Vorbereitung zu diesem Feldzug besteht unter anderem darin, einen Waffenschmied zur Herstellung eines Schwertes zu überreden. Fanni verwendet, davon inspiriert, viel Zeit und Aufwand darauf, in einer verlassenen ehemaligen Sensenfabrik ein Sensenblatt in ein Katana umzuwandeln (MH 178).

Die Anwendung des Katana findet jedoch nicht statt - bloß in einer fiktiven Binnenerzählung (aber auch Vorstellungen sind »eine ziemlich indikativische Angelegenheit«, wie der Ire aus der Kurzen Geschichte einwerfen würde - KG 31). Fanni selbst erdichtet Geschichten, die sie aufschreibt und von denen sie einige ihrer Adoptivschwester Switi erzählt. Darunter findet sich auch eine, in der sie die Ermordung ihres Adoptivvaters (»den ich jetzt Bill nenne« - MH 115) imaginiert. Auch die Handlungsabfolge dieser Tat entspricht in groben Zügen einer Szene aus Kill 
Bill (MH 286): Ein Mädchen verführt und ermordet darin einen pädophilen Unterweltboss. ${ }^{6}$

Die Figurenkonstellationen beider Racheakte in Kill Bill beinhalten die skandalöse Verbindung einer sexualisierten und gewaltsamen Beziehung zu einem Kind: Im einen Fall über den realen Kindesmissbrauch, im anderen assoziativ über einen Kosenamen (die Protagonistin wird von Bill >Kiddo< gerufen). Daraus ergibt sich jedenfalls auf Figurenebene des Romans ausreichend Identifikationspotenzial für Fanni.

Dennoch gibt es für einen von ihren populärkulturellen Vorbildern inspirierten Mord am Adoptivvater keine Belege im Text. Fanni lässt stattdessen ihrer Lehrerin die DVD zukommen, auf der ihr Stiefvater die Ermordung ihrer Adoptivschwester festgehalten hat, und deckt so das Verbrechen auf. Übergeben wird die DVD in der Hülle des Tarantino-Films, die Wahrheit kommt also ganz konkret sim Gewand der Fiktion ans Licht.

Warum jedoch wird das Schwert, aller motivischen und handwerklichen Vorbereitung zum Trotz, nicht tatsächlich verwendet? Es scheint eine andere Funktion (eine Art Relaisfunktion) zu haben: Es dient, indem es zugleich in der Rachephantasie der fiktionalen Binnenerzählung und auf der Handlungsebene erscheint, als materieller Bezugspunkt, als paradoxer materieller Garant für den Möglichkeitsraum, den auch eine fiktive Tat eröffnet.

Dominiks Revolver ermöglicht die rein symbolische Verabschiedung der Väter, Fannis Schwert eine Rache, die schließlich ohne dieses Schwert durchgeführt wird. Der sprachliche und kulturelle Kontext der Waffen, ihre Herkunft aus kollektiv hergestellten und rezipierten Imaginationen weist auf einen intrikaten Punkt psychoanalytischer Fetischtheorien hin: dass die Arsenale des Fetischismus stets der Kultur entnommen, also immer schon kulturell codiert sind. Dieser Punkt ist natürlich wiederum Kern derjenigen Fetischkonzepte, wie sie von kulturkritischer Seite formuliert wurden. Allerdings sind hier Protagonistin und Protagonist nicht bloß passive Empfänger kultureller Inhalte, wie man aus dieser Perspektive monieren und dabei etwa die Rede von der >Kulturindustrie` ins Feld führen könnte: Aus der Aneignung und der darauf folgenden Anverwandlung der kollektiven Imagination erhalten die Dinge ihr Potenzial zur Ermächtigung des Subjekts (ganz im Sinne et-

6 Anzumerken sind einige Abweichungen von der >Filmvorlage : Die Schwertführung Fannis nämlich entspricht nicht der O-Rens (die anscheinend gezielt durch die Bauchdecke ein großes Blutgefäß durchstößt), stattdessen finden sich hier Anleihen an das japanische Seppuku (MH 173). Überhaupt ist die Selbststilisierung Fannis ein kultureller Hybrid: Das Ritual ist fernöstlich inspiriert, die rituelle Bekleidung liefert hingegen das katholische Ordenswesen (die Cuculla); das fernöstliche Katana entsteht aus einem westlichen Sensenblatt. 
168 Üble Dinge. Materialität und Fetischismus in der Prosa Paulus Hochgatterers

wa Fiskes) ${ }^{7}$. Dem individuellen Fortspinnen kultureller Inhalte - paradigmatisch etwa in den erzählenden Künsten - wird ein emanzipatorisches Moment attestiert. Gerade die so suspekte Ding-Kategorie der Waffe hält damit einen emphatischen poetologischen Kommentar bereit.

7 Vgl. dazu die Stellung der Popularkultur bei John Fiske: ders., Popularkultur verstehen (2003 [1989]). 